\title{
AID, p53 and MLH1 expression in early gastric neoplasms and the correlation with the background mucosa
}

\author{
SOICHIRO KAWATA, KAZUO YASHIMA, SOHEI YAMAMOTO, SHUJI SASAKI, YOHEI TAKEDA, \\ AKIHIRO HAYASHI, KAZUYA MATSUMOTO, KOICHIRO KAWAGUCHI, \\ KENICHI HARADA and YOSHIKAZU MURAWAKI
}

Division of Medicine and Clinical Science, Faculty of Medicine, Tottori University, Nishicho, Yonago 683-8504, Japan

Received August 6, 2014; Accepted April 13, 2015

DOI: $10.3892 / 01.2015 .3342$

\begin{abstract}
A number of tumor-associated genes have been associated with gastric cancer development. The present study evaluated differences in tumor-associated protein expression and phenotype among early gastric neoplasms, and correlated these data with those of the background mucosa. The expression of activation-induced cytidine deaminase (AID), p53 and MLH1 in 151 early gastric neoplasms [22 gastric adenomas, 92 intramucosal carcinomas (MCs), and 37 submucosal carcinomas (SMCs)] was examined immunohistochemically and compared with that of the corresponding background mucosal condition. The cellular phenotypes of the neoplasms and the corresponding background intestinal metaplasia were also determined. Aberrant AID, p53 and MLH1 expression was detected in 36.4, 0 and $0 \%$ of the adenomas, in $35.9,32.6$ and $16.3 \%$ of the MCs, and in 56.8, 62.2 and $21.6 \%$ of the SMCs, respectively. The frequency of aberrant AID and p53 expression in the SMCs was significantly increased compared with that in the MCs (AID, $\mathrm{P}<0.05$; p53, $\mathrm{P}<0.01$ ). Aberrant AID expression was significantly associated with $\mathrm{p} 53$ overexpression in the SMCs $(\mathrm{P}<0.01)$, but not in the adenomas or MCs. In addition, AID expression was associated with the severity of mononuclear cell activity in the non-cancerous mucosa adjacent to the tumor $(\mathrm{P}<0.05)$, particularly in the SMC cases. The percentage of MCs (34.8\%) and SMCs (24.3\%) that were of the gastric phenotype was higher compared with the percentage of adenomas (18.2\%). These results indicated that p53 and MLH1 expression and a gastric phenotype may be important for carcinogenesis, and that chronic inflammation and AID and p53 expression are associated with submucosal progression.
\end{abstract}

Correspondence to: Dr Kazuo Yashima, Division of Medicine and Clinical Science, Faculty of Medicine, Tottori University, 36-1 Nishicho, Yonago 683-8504, Japan

E-mail: yashima@grape.med.tottori-u.ac.jp

Key words: gastric cancer, endoscopic resection, AID, p53, phenotype

\section{Introduction}

Despite the worldwide reduction in gastric cancer mortality in the last decade, the disease remains a notable health problem (1). Helicobacter pylori (HP) infection results in gastric cancer through the slow progression of the premalignant stages of intestinal metaplasia (IM), atrophic gastritis and adenoma/dysplasia (2). In addition, a number of previous studies have indicated the possibility that highly active inflammation induced by HP is involved in cancer development $(3,4)$. However, the molecular mechanisms that underlie gastric cancer development through the progression of HP-associated chronic gastritis have not been well studied.

Gastric cancer results from the accumulation of genetic and epigenetic alterations (5). In sporadic gastric cancer, the frequency of p53 gene mutations is $25-50 \%(5,6)$ and the frequency of DNA methylation of the DNA mismatch repair gene mutL homolog 1 (MLH1) gene is $20-30 \%(5,7)$. Hypermethylation of the $M L H 1$ promoter region is the primary cause of microsatellite instability in primary gastric cancer (8). Previous studies have also demonstrated that activation-induced cytidine deaminase (AID) is important in HP-associated gastric cancer development (9). AID is an enzyme that edits DNA and RNA, and was originally identified to induce somatic hypermutations and class-switch recombination in immunoglobulin genes (10). Previous studies have indicated that AID transgenic mice develop malignant T-cell lymphomas and lung adenomas. These findings indicate that aberrant AID expression results in tumor-associated gene mutations and may be a cause of human malignancy (11). Cag-pathogenicity island (cag-PAI)-positive HP infection leads to the activation of nuclear factor (NF)- $\kappa \mathrm{B}$ in the gastric epithelium. As a result, AID is transcriptionally upregulated in gastric epithelial cells, and may contribute to the accumulation of $p 53$ and other gene mutations, leading to gastric carcinogenesis (12). However, the histological association between AID expression, gastric tumors and the background mucosa is not well understood.

Gastric cancer has been divided into two histological types: Intestinal and diffuse [Lauren's classification (13)] or differentiated and undifferentiated [Nakamura et al (14)]. Previous studies using mucin-based histochemical and immunohistochemical analyses indicated that gastric and intestinal 
phenotypic cell markers are widely expressed in gastric cancer, irrespective of the histological type (15-17).

In the present study, to improve the understanding of the mechanisms underlying malignant transformation in gastric cancer and its association with developmental processes, the expression of AID, p53 and MLH1, and the phenotypic expression in early gastric neoplasms were evaluated and compared with the background mucosal condition.

\section{Patients and methods}

Patient and tissue samples. Gastric tumors were obtained from 151 patients (109 males and 42 females) who had undergone endoscopic or surgical tumor resection at the Tottori University Hospital between 2003 and 2009, and who possessed histopathologically diagnosed tubular adenomas ( $\mathrm{n}=22$; males, 14; females, 8; average age, 72.5 years), intramucosal carcinomas (MCs; $n=92$; males, 67; females, 25; average age, 70.2 years), and submucosal carcinomas (SMCs; $\mathrm{n}=37$; males, 28; females, 9; average age, 66.8 years), according to the Japanese Classification of Gastric Carcinoma (Table I) (18). All tubular adenomas corresponded to category 3, all MCs were classified as category 4 and all SMCs as category 5 of the Vienna classification (19). The mean age was statistically younger in the SMC group compared with the adenoma and MC groups. A younger age, larger tumor size and depressed type tumors were more frequently observed in the patients with SMCs compared with those with adenomas and MCs.

All patients were classified as having HP infections based on endoscopic atrophic changes or by testing seropositive for HP-immunoglobulin G (IgG). It was further confirmed that these patients had no HP eradication history. All specimens were assigned a novel number without the inclusion of personal information in order to maintain anonymity. This study was approved by the Institutional Ethics Committee of Tottori University (no. 314) and complies with the Declaration of Helsinki.

Evaluation of the tumor-surrounding mucosa. HP-associated gastritis adjacent to the tumor was evaluated by using the updated Sydney system (USS) (20). Using this system, gastritis was scored from 0 (absent) to 3 (marked). Glandular atrophy, IM and mononuclear cell infiltration (chronic inflammation) were examined. IM adjacent to the tumor was absent in 1 adenoma, $1 \mathrm{MC}$ and $6 \mathrm{SMC}$ cases.

Immunohistochemical staining. Paraffin-embedded sections (4- $\mu \mathrm{m}$ thick) were immunohistochemically stained with the indicated mouse monoclonal antibodies against the following proteins: p53 (DO-7; Dako, Glostrup, Denmark; dilution, 1:50), MutL homolog 1 (MLH1; G168-15; BD Pharmingen, San Diego, CA, USA; dilution, 1:50), mucin 5AC, oligomeric mucus/gel-forming (MUC5AC; 45M1; Leica Biosystems, Ltd., Newcastle, UK; dilution, 1:50), mucin 2, oligomeric mucus/gel-form (MUC2; Ccp58; Leica Biosystems, Ltd.; dilution, 1:100) and cluster of differentiation 10 (CD10; 56C6; Leica Biosystems, Ltd.; dilution, 1:50) or with a rat monoclonal anti-AID antibody (EK2 5G9; Cell Signaling Technology, Inc., Danvers, CA,
USA; dilution 1:400), using the avidin-biotin-peroxidase complex (ABC) technique.

Immunohistochemical staining was performed in a blinded manner with respect to the clinical information. The sections were deparaffinized in xylene and rehydrated in ethanol. The sections were then immersed in a citrate buffer $(0.01 \mathrm{~mol} / \mathrm{l}$; $\mathrm{pH}$ 6.0) and heated in a microwave oven for 20-30 $\mathrm{min}$ to retrieve antigens. Endogenous tissue peroxidase activity was blocked by incubation with $3 \% \mathrm{H}_{2} \mathrm{O}_{2}$. The sections were subsequently incubated with primary antibody overnight at $4^{\circ} \mathrm{C}$. As a negative control, the primary antibody was replaced with normal serum $\operatorname{IgG}$ at a similar dilution. Signal detection followed the Vectastain Elite ABC kit instructions (Vector Laboratories, Inc., Burlingame, CA, USA) using diaminobenzidine as the chromogen. The sections were counterstained with hematoxylin, and were subsequently incubated with biotinylated anti-rat or anti-mouse $\mathrm{IgG}$ and avidin-biotin-peroxidase (included with Vectastatin Elite ABC Kit). The sections were subsequently visualized using diaminobenzidine tetrahydrochloride. Two independent observers evaluated protein expression using an optical microscope (BX50; Olympus Corporation, Tokyo, Japan).

Assessment of AID immunostaining. The internal positive controls were lymphocytes of germinal centers in lymphoid follicles (Fig. 1A). The follicles contain activated B cells and all specimens stained markedly positive for AID. The cytoplasm was scored as positive when $>30 \%$ of the tumor cells stained as markedly as the germinal centers. Aberrant AID expression refers to AID overexpression.

Assessment of p53 immunostaining. The tumors were scored as positive for $\mathrm{p} 53$ when a distinct nuclear immunoreaction occurred in $>25 \%$ of the tumor cells (21), as presented in Fig. 1B. Positive staining for p53 is considered aberrant p53 expression (overexpression).

Assessment of MLH1 immunostaining. MLH1 expression was classified as either normal or reduced (aberrant). Tissue specimens with definite nuclear staining in $<30 \%$ of the tumor cells were categorized as having reduced staining (22).

Assessment of MUC5AC, MUC2 and CD10 immunostaining, and classification of phenotypes. Staining of $>10 \%$ of the adenoma and carcinoma cells for MUC5AC, MUC2 or CD10 was classified as positive for that marker, and staining of $<10 \%$ was classified as negative. The tumor phenotypes were classified into 3 categories according to the combination of the expression of CD10, MUC2 and MUC5AC. The intestinal phenotype (I-type) was positive for MUC2 and/or CD10, but negative for MUC5AC. The gastric and intestinal mixed phenotype (GI-type) was positive for MUC2 and/or CD10, and positive for MUC5AC. The gastric phenotype (G-type) was positive for MUC5AC, but negative for MUC2 and CD10. IM was also classified into GI- and I-types using these markers (23).

Statistical analysis. All data were statistically analyzed using the $\chi^{2}$ test with Yates' correction, Fisher's test and Student's $t$-test using Stat View software, version 5.0 (SAS Institute, 
Table I. Clinicopathological characteristics of early gastric neoplasias.

\begin{tabular}{|c|c|c|c|}
\hline Characteristic & Adenoma $(n=22)$ & $\mathrm{MC}(\mathrm{n}=92)$ & $\mathrm{SMC}(\mathrm{n}=37)$ \\
\hline Age, years (mean $\pm S D)$ & $72.5 \pm 9.2$ & $70.2 \pm 7.4$ & $66.8 \pm 12.2$ \\
\hline \multicolumn{4}{|l|}{ Gender, $\mathrm{n}$} \\
\hline Male & 14 & 67 & 28 \\
\hline Female & 8 & 25 & 9 \\
\hline Size, mm (mean \pm SD) & $11.6 \pm 7.2$ & $10.0 \pm 4.5$ & $25.3 \pm 14.9$ \\
\hline \multicolumn{4}{|l|}{ Site, $\mathrm{n}$} \\
\hline Upper third & 4 & 19 & 6 \\
\hline Middle third & 6 & 38 & 20 \\
\hline Lower third & 12 & 35 & 11 \\
\hline \multicolumn{4}{|l|}{ Macroscopic type, $n$} \\
\hline Elevated & 11 & 31 & 4 \\
\hline Flat or depressed & 11 & 61 & 33 \\
\hline \multicolumn{4}{|l|}{ Histology, n } \\
\hline Differentiated & & 91 & 23 \\
\hline Mixed & NA & 1 & 5 \\
\hline Undifferentiated & & 0 & 9 \\
\hline
\end{tabular}

SD, standard deviation; NA, not applicable; MC, intramucosal carcinoma; SMC, submucosal carcinoma. 'Differentiated' includes tubular and papillary types. 'Undifferentiated' consists of signet-ring cell and/or poorly-differentiated types. 'Mixed' is composed of 'Differentiated' and 'Undifferentiated'.

Table II. Percentages of early gastric neoplasias showing aberrant expression of AID, p53 and MLH1.

\begin{tabular}{lcrr}
\hline & & Aberrant expression, n/total n $(\%)$ & SMC (n=37) \\
\cline { 2 - 4 } Protein & Adenoma $(\mathrm{n}=22)$ & MC (n=92) & $21 / 37(56.8)^{\mathrm{a}}$ \\
\hline AID & $8 / 22(36.4)$ & $33 / 92(35.9)^{\mathrm{a}}$ & $23 / 37(62.2)^{\mathrm{b}}$ \\
p53 & $0 / 22(0.0)$ & $30 / 92(32.6)^{\mathrm{b}}$ & $8 / 37(21.6)$ \\
MLH1 & $0 / 22(0.0)$ & $15 / 92(16.3)$ & S \\
\hline
\end{tabular}

${ }^{\mathrm{a}} \mathrm{P}=0.048,{ }^{\mathrm{b}} \mathrm{P}=0.004$. AID, activation-induced cytidine deaminase; MLH1, MutL homolog 1; MC, intramucosal carcinoma; SMC, submucosal carcinoma.

Cary, NC, USA). P<0.05 was considered to indicate a statistically significant difference.

\section{Results}

Frequency of aberrant AID, p53 and MLH1 expression. The protein expression of AID, p53 and MLH1 was immunohistochemically determined in early gastric neoplasms (Table II). Aberrant AID, p53 and MLH1 expression (overexpression, overexpression and loss of expression, respectively) was detected in $36.4 \%(8 / 22), 0 \%(0 / 22)$ and $0 \%(0 / 22)$ of the adenomas, in $35.9 \%$ (33/92), 32.6\% (30/92) and $16.3 \%$ (15/92) of the MCs, and in 56.8\% (21/37), 62.2\% (23/37) and $21.6 \%(8 / 37)$ of the SMCs, respectively. p53 overexpression and loss of MLH1 expression was not detected in the adenomas. Overexpression of AID and the p53 gene product was significantly more frequent in the SMCs compared with the MCs (AID, $\mathrm{P}<0.05$; $\mathrm{p} 53, \mathrm{P}<0.01)$. There were no significant differences in the loss of MLH1 between the MCs and SMCs. In addition, there were no significant correlations between the expression levels of these proteins and other clinicopathological data, including age, gender, location or macroscopic type of tumor (data not shown).

Association between AID, p53 and MLH1 expression. Aberrant AID expression was significantly associated with p53 overexpression in the SMCs $(\mathrm{P}<0.01)$, but not in the MCs (Table III). p53 overexpression was significantly and inversely associated with the loss of MLH1 expression, particularly in the MCs $(\mathrm{P}<0.05)$. The percentage of SMCs that demonstrated aberrant AID and p53 expression (45.9\%) was significantly increased compared with that of the MCs $(10.9 \%)(\mathrm{P}<0.0001)$. When the expression of aberrant AID and p53 was used for the diagnosis of submucosal invasion, the sensitivity was $45.9 \%$, the 
Table III. Association between AID and p53 expression in early gastric cancers.

\begin{tabular}{|c|c|c|c|c|c|c|}
\hline \multirow[b]{3}{*}{ p53 expression } & \multicolumn{6}{|c|}{ AID expression } \\
\hline & \multicolumn{2}{|c|}{$\mathrm{MC}(\mathrm{n}=92)$} & \multirow[b]{2}{*}{ P-value } & \multicolumn{2}{|c|}{ SMC $(n=37)$} & \multirow[b]{2}{*}{ P-value } \\
\hline & Positive, $\mathrm{n}$ & Negative, $\mathrm{n}$ & & Positive, $\mathrm{n}$ & Negative, $\mathrm{n}$ & \\
\hline Positive & 10 & 20 & & 17 & 6 & \\
\hline Negative & 23 & 39 & $\mathrm{P}=0.724$ & 4 & 10 & $\mathrm{P}=0.0069$ \\
\hline
\end{tabular}

AID, activation-induced cytidine deaminase; MC, intramucosal carcinoma; SMC, submucosal carcinoma.

Table IV. Association between AID and p53 expression and submucosal invasion in early gastric cancers.

\begin{tabular}{lcccc}
\hline $\begin{array}{l}\text { Aberrant } \\
\text { expression }\end{array}$ & $\begin{array}{c}\text { MCs }+ \\
\text { SMCs }\end{array}$ & $\begin{array}{c}\text { MC } \\
(\mathrm{n}=92)\end{array}$ & $\begin{array}{c}\text { SMC } \\
(\mathrm{n}=37)\end{array}$ & P-value \\
\hline AID(+)/p53(+), n & 27 & 10 & 17 & \\
Other, $\mathrm{n}$ & 102 & 82 & 20 & $\mathrm{P}<0.0001$ \\
\hline
\end{tabular}

Sensitivity, $45.9 \%$ (17/37); specificity, $89.1 \%$ (82/92); positive predictive value, $63.0 \%$ (17/27); negative predictive value, $80.4 \%(82 / 102)$. AID, activation-induced cytidine deaminase; $\mathrm{MC}$, intramucosal carcinoma; SMC, submucosal carcinoma.

Table V. Updated Sydney system score of the background mucosa of early gastric neoplasias.

\begin{tabular}{lccc}
\hline Parameter & $\begin{array}{c}\text { Adenoma } \\
(\mathrm{n}=22)\end{array}$ & $\begin{array}{c}\text { MC } \\
(\mathrm{n}=92)\end{array}$ & $\begin{array}{c}\text { SMC } \\
(\mathrm{n}=37)\end{array}$ \\
\hline Atrophy & $2.22^{\mathrm{a}}$ & 1.83 & 1.89 \\
IM & $2.31^{\mathrm{b}}$ & 1.90 & 1.78 \\
Chronic inflammation & 1.77 & 1.89 & $2.24^{\mathrm{c}}$ \\
\hline
\end{tabular}

a,b Mean scores of glandular atrophy and IM surrounding the adenomas were significantly higher than those surrounding the MCs and SMCs $(\mathrm{P}<0.05)$. ${ }^{\circ}$ The mean score of chronic inflammation surrounding the SMCs was higher than those surrounding the adenomas and MCs $(\mathrm{P}<0.01)$. MC, intramucosal carcinoma; SMC, submucosal carcinoma; IM, intestinal metaplasia.

specificity was $89.1 \%$, the positive predictive value was $63.0 \%$ and the negative predictive value was $80.4 \%$ (Table IV).

Association between AID, p53 and MLH1 expression levels, and the background mucosa. Next, the gastritis in the background mucosa of the tumors was analyzed by evaluating the glandular atrophy, IM and chronic inflammation (mononuclear cell infiltration). Table $\mathrm{V}$ shows the mean USS scores for these parameters. The mean scores of gastritis surrounding the adenomas, MCs and SMCs were as follows: Glandular atrophy, 2.22, 1.83 and 1.89; chronic inflammation, 1.77, 1.89 and 2.24; and IM, 2.31, 1.90 and 1.78, respectively. A significant increase was noted in the mean scores of glandular
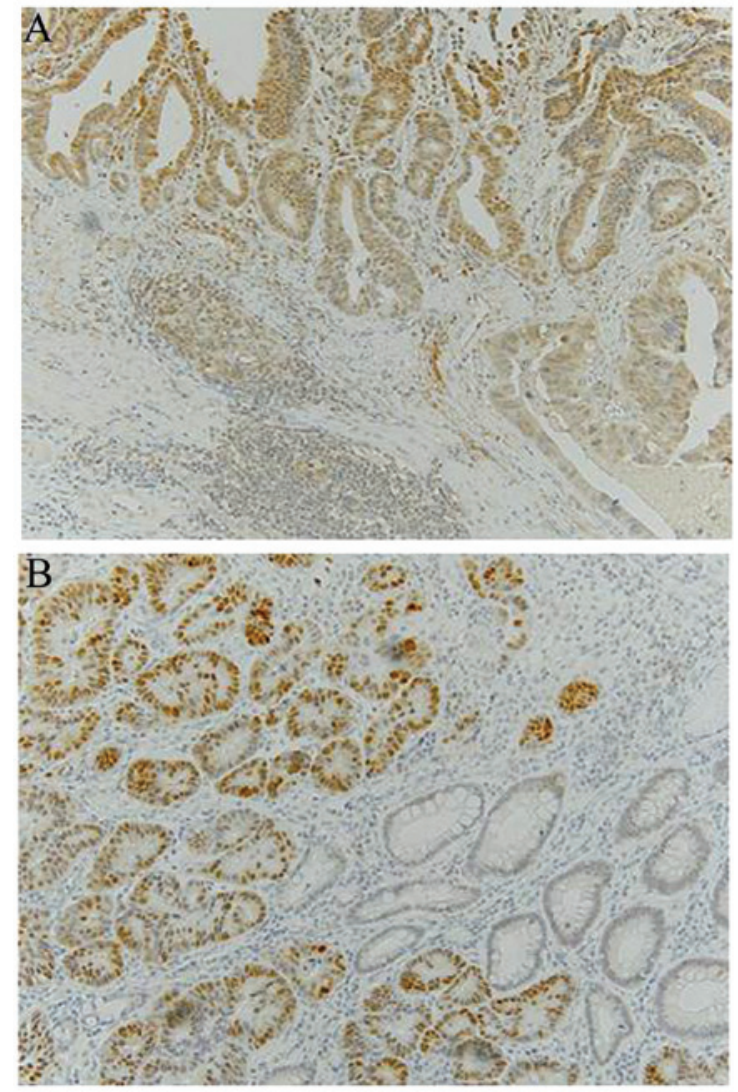

Figure 1. Representative images of AID and p53 immunostaining in an early gastric neoplasm. (A) Positive AID immunostaining in the cytoplasm of intramucosal carcinoma. (B) Overexpression of p53 in intramucosal carcinoma. AID, activation-induced cytidine deaminase.

atrophy and IM surrounding the adenomas compared with those surrounding the MCs and SMCs $(\mathrm{P}<0.05)$, and the mean score of chronic inflammation surrounding the SMCs was significantly increased compared with those surrounding the adenomas and MCs $(\mathrm{P}<0.01)$. The mean score of chronic inflammation in the AID-positive neoplasms was significantly increased compared with that in the AID-negative neoplasms, particularly in the SMC cases $(\mathrm{P}<0.05)$ (Fig. 2A). In addition, the mean score of chronic inflammation in the MLH1-negative neoplasms was reduced compared with the MLH1-positive neoplasms $(\mathrm{P}<0.05$; Fig. $2 \mathrm{~B})$. The mean score of IM adjacent to the p53-positive SMCs was increased compared with that adjacent to the p53-negative SMCs (Fig. 2C). 
Table VI. Phenotype of early gastric neoplasias and their surrounding IM.

\begin{tabular}{lcrr}
\hline Parameter & Adenoma $(\mathrm{n}=22)$ & MC $(\mathrm{n}=92)$ & $\mathrm{SMC}(\mathrm{n}=37)$ \\
\hline Phenotype of tumor, $\mathrm{n}(\%)$ & & & \\
G-type & $4(18.2)$ & $32(34.8)$ & $9(24.3)$ \\
GI-type & $6(27.3)$ & $33(35.9)$ & $16(43.2)$ \\
I-type & $12(54.5)$ & $27(29.3)$ & $12(32.4)$ \\
Phenotype of surrounding IM, n (\%) & & & \\
GI-type & $15(71.4)$ & $66(72.5)$ & $17(54.8)$ \\
I-type & $6(28.6)$ & $25(27.5)$ & $14(45.2)$ \\
\hline
\end{tabular}

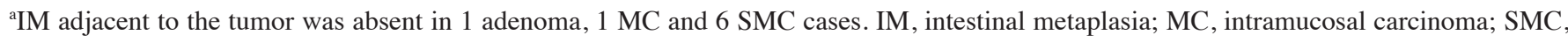
submucosal carcinoma; G-type, gastric phenotype; GI-type, gastric and intestinal mixed phenotype; I-type, intestinal phenotype.

Phenotype of tumors and their surrounding IM. The gastric/intestinal phenotypes of the tumors and their surrounding IM were analyzed (Table VI). A high percentage of MCs (34.8\%) and SMCs (24.3\%) were of the G-type, and a high percentage of adenomas $(54.5 \%)$ were of the I-type $(\mathrm{P}<0.05)$. Using the same phenotypic markers, the tumor-surrounding IM was divided into two major types: A GI-type and a solely I-type. The phenotype of the IM surrounding SMCs was largely classified as the I-type (45.2\%), and the phenotypes of the IMs surrounding the adenomas and the majority of the MCs were classified as the GI-type (71.4 and $72.5 \%$, respectively; $\mathrm{P}=0.063$ ). The combined data indicated that the background IM phenotype was similar for the adenomas and MCs, but differed for the SMCs. In addition, the cellular phenotype in tumors and the background mucosa were not correlated with tumor-associated protein expression or with the mean scores of the USS classification (data not shown).

\section{Discussion}

The present study examined AID, p53 and MLH1 expression, and the cellular phenotype of early gastric neoplastic samples, and then these results were compared with patient clinicopathological characteristics and the condition of the mucosa surrounding or adjacent to the tumor. The expression levels of p53 and MLH1, and a gastric phenotype were significantly associated with carcinogenesis. In addition, the features of the background mucosa of the adenomas and MCs were similar. Notably, chronic inflammation and AID and p53 expression were significantly associated with submucosal invasion.

Previous studies have demonstrated important roles for AID, a cellular genome mutator, in HP-associated gastric cancer development (9). Infection with HP triggers the aberrant expression of AID within the gastric epithelium, which results in the accumulation of altered nucleotides in the p53 gene $(12,24,25)$. The percentage of adenomas $(36.4 \%)$, MCs $(35.9 \%)$ and SMCs $(56.8 \%)$ that demonstrated aberrant AID expression in the present study was slightly increased compared with the percentage of GCs that demonstrated aberrant AID expression in two previous studies (26.9 and $22.5 \%)(25,26)$. Notably, in the present study, the percentage of adenomas and MCs that demonstrated aberrant AID
A

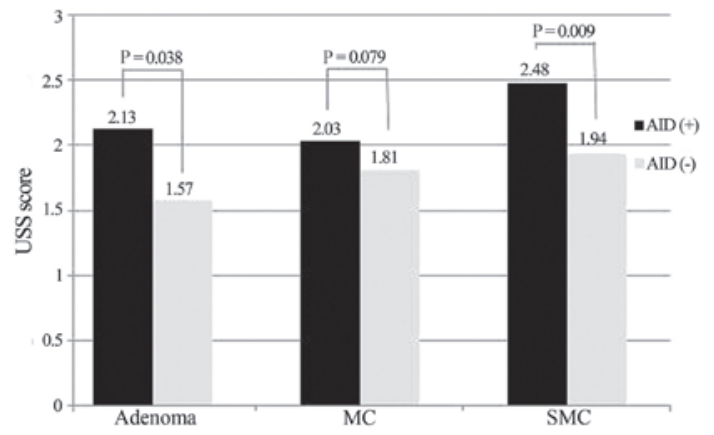

B

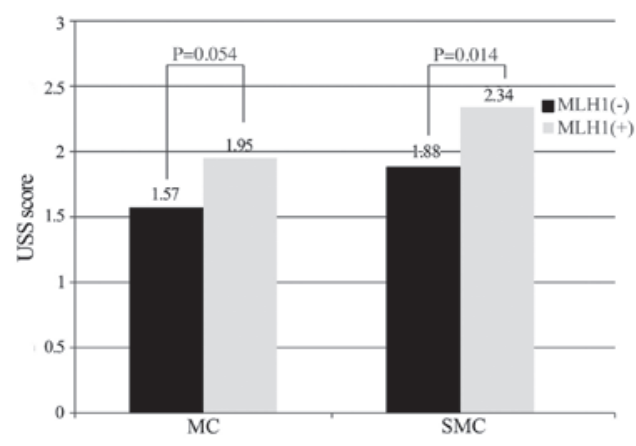

C

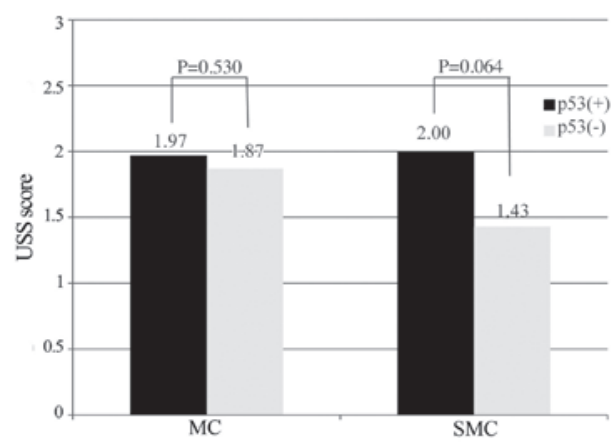

Figure 2. Expression of (A) AID, (B) MLH1 and (C) p53 in early gastric neoplasias and USS scores of (A and B) chronic inflammation and (C) IM in their background mucosa. (A) The mean score of chronic inflammation in the AID-positive neoplasms was significantly increased compared with that in the AID-negative neoplasms, particularly in the SMC cases $(\mathrm{P}<0.05)$. (B) The mean score of chronic inflammation in MLH1-negative neoplasms tended to be low compared to MLH1-positive neoplasms $(\mathrm{P}<0.05)$. (C) The mean score of IM adjacent to p53-positive SMCs tended to be high compared with that adjacent to p53-negative SMCs. AID, activation-induced cytidine deaminase; MLH1, MutL homolog 1; USS, updated Sydney system; MC, intramucosal carcinoma; SMC, submucosal carcinoma; IM, intestinal metaplasia. 
expression was the same, whereas the percentage of SMCs demonstrating aberrant AID expression was significantly increased compared with that of MCs. The variability in the findings may be a result of the differences in the stage of carcinoma progression and in the degree of tumor differentiation.

Kim et al (25) observed a significant association between the aberrant expression of AID and the nuclear overexpression of p53 in a range of gastric cancer types. However, our previous study did not demonstrate an association between aberrant AID expression and p53 overexpression in early differentiated gastric cancer (26). Similarly, Goto et al (27) observed no correlation between AID and p53 expression in early differentiated and poorly-differentiated gastric cancer. In the present study, aberrant AID expression was significantly associated with $\mathrm{p} 53$ overexpression in the SMCs $(\mathrm{P}<0.01)$, but not in the MCs or adenomas. Therefore, mutations in p53 as a result of aberrant AID expression may increase with tumor progression. However, the p53 protein may also become altered through cigarette smoking, as occurs in lung and esophageal carcinogenesis $(28,29)$. AID is a well-described molecule that is involved in DNA mutations in the human genome. The typical pattern of nucleotide alteration induced by its enzymatic activity is cytosine/guanine $(\mathrm{C} / \mathrm{G})$ to thymine/adenine (T/A) transition (9). Tobacco smoking also induces G/C to T/A transversion (30). Further investigation is required to clarify the correlation between the expression of p53 and aberrant AID expression.

The expression of AID in gastric epithelial cells may be altered by the direct action of HP macromolecules through the type IV secretion system encoded by cag-PAI (31). Additionally, in human gastric epithelial cells, AID expression is induced by HP-associated inflammatory mediators, including tumor necrosis factor $\alpha$ and interleukin- $\beta$ via activation of $\mathrm{NF}-\kappa \mathrm{B}$ (12). Furthermore, the expression of AID in tumors, including colon cancer, cholangiocarcinoma and hepatocellular carcinoma, is also mediated by stimulation of proinflammatory cytokines via NF- $\kappa \mathrm{B}(32-34)$. Aberrant AID expression correlates with chronic active inflammation, glandular atrophy and IM in non-neoplastic gastric mucosa (26). The present study demonstrated that aberrant AID expression in tumors correlated with mononuclear cell activity in the mucosa surrounding the tumor, particularly in SMC cases, which supports the aforementioned mechanism of AID expression and is consistent with previous studies $(26,27)$. Nagata et al (35) demonstrated that the enhanced expression of AID in human gastric mucosa infected by HP is reduced following HP eradication. In addition, Kang et al (36) reported that rebamipide suppressed HP-induced AID expression and also suppressed invasion of gastric cancer cells through the downregulation of phospholipase D1 (PLD1), via inhibition of the HP cagA-NFKB-PLD1 signaling pathway. Therefore, HP eradication and a drug that results in AID suppression via inhibition of HP-mediated PLD1 may prevent submucosal invasion in gastric cancer.

Submucosal invasion in gastric cancer has also been associated with aberrant AID and p53 expression. Sugai et al (37) reported that gastric cancer with p53 overexpression is associated with a high risk of submucosal invasion. These data indicate that AID is important in the progression of gastritis to gastric cancer, particularly in SMC. The results of the present study indicated that tumor progression may be associated with p53 mutations that arise due to aberrant AID expression. Also, combined aberrant AID expression and p53 overexpression may provide valuable information for the prediction of aggressive tumor behavior.

In the present study, the percentage of the MCs and SMCs that demonstrated loss of MLH1 expression was 16.3 and $21.6 \%$, respectively, and this loss of MLH1 expression was inversely associated with p53 overexpression in the MCs. These findings are consistent with previous studies (5). Aberrant DNA methylation of promoter $\mathrm{CpG}$ islands appears to be the predominant mechanism associated with the loss of $h M L H 1$ function in chronic inflammation-associated sporadic gastric carcinomas (8). In addition, previous studies have demonstrated that AID is crucial in inducing $p 53$ mutations and DNA demethylation (38). This mechanism may explain this association. In addition, in the present study there was no difference in MLH1 expression between MCs and SMCs, indicating that loss of MLH1 expression appears to be independent of the degree of invasion, which is consistent with previous study findings (37).

Marked differences between the biological behavior of gastric phenotype carcinomas and intestinal phenotype carcinomas have previously been reported (39). In the present study, the percentage of the MCs (34.8\%) and SMCs (24.3\%) that were of the gastric phenotype was significantly increased compared with that of the adenomas (18.2\%), and the percentage of the adenomas that were of the intestinal phenotype $(54.5 \%)$ was increased compared with that of the MCs and SMCs (29.3 and 32.4\%). Based on these data, the gastric phenotype may be important for malignant transformation. This result is consistent with previous studies (40). The current study further demonstrated that the phenotype of the background IM of the SMCs was largely classified as the intestinal type (45.2\%), whereas the majority of the phenotypes of the background IMs of the adenomas and MCs were classified as the mixed type (71.4 and $72.5 \%$ respectively). The combined data indicated that the background IM phenotype of the adenomas and MCs was similar, but that the phenotype of the SMCs was different. Previous observations in animal models indicated that the phenotype of IM changed from the GI mixed-type to type I during tumor progression (41). This may indicate a phenotypic shift in expression from the gastric to intestinal phenotype during tumor progression.

In conclusion, p53 and MLH1 expression, and a gastric phenotype may be important for carcinogenesis, and adenomas and MCs may arise from a similar background mucosa. Furthermore, chronic inflammation and AID and p53 expression may affect submucosal invasion. These findings may provide valuable information for the prediction of aggressive tumor behavior and may aid in the decision between endoscopic therapies and surgical resection. However, additional extensive future studies are required to confirm the results of the present study.

\section{Acknowledgements}

This study has been presented as a 2013 United European Gastroenterology Week oral presentation. 


\section{References}

1. Siegel R, Naishadham D and Jemal A: Cancer statistics, 2012. CA Cancer J Clin 62: 10-29, 2012.

2. Correa P: Human gastric carcinogenesis: A multistep and multifactorial process - First American cancer society award lecture on cancer epidemiology and prevention. Cancer Res 52: 6735-6740, 1992.

3. Uemura N, Okamoto S, Yamamoto S, et al: Helicobacter pylori infection and the development of gastric cancer. N Engl J Med 345: 784-789, 2001.

4. Sipponen P and Kimura K: Intestinal metaplasia, atrophic gastritis and stomach cancer: Trends over time. Eur J Gastroenterol Hepatol 6 (Suppl 1): S79-S83, 1994.

5. Tamura G: Alterations of tumor suppressor and tumor-related genes in the development and progression of gastric cancer. World J Gastroenterol 12: 192-198, 2006.

6. Yamazaki K, Tajima Y, Makino R, et al: Tumor differentiation phenotype in gastric differentiated-type tumors and its relation to tumor invasion and genetic alterations. World J Gastroenterol 12 : 3803-3809, 2006.

7. Hong SH, Kim HG, Chung WB, et al: DNA hypermethylation of tumor-related genes in gastric carcinoma. J Korean Med Sci 20 : 236-241, 2005.

8. Fleisher AS, Esteller M, Wang S, et al: Hypermethylation of the hMLH1 gene promoter in human gastric cancers with microsatellite instability. Cancer Res 59: 1090-1095, 1999.

9. Chiba T, Marusawa $\mathrm{H}$ and Ushijima T: Inflammation-associated cancer development in digestive organs: Mechanisms and roles for genetic and epigenetic modulation. Gastroenterology 143: 550-563, 2012.

10. Muramatsu M, Kinoshita K, Fagarasan S, Yamada S, Shinkai Y and Honjo T: Class switch recombination and hypermutation require activation-induced cytidine deaminase (AID), a potential RNA editing enzyme. Cell 102: 553-563, 2000.

11. Okazaki IM, Hiai H, Kakazu N, et al: Constitutive expression of AID leads to tumorigenesis. J Exp Med 197: 1173-1181, 2003

12. Matsumoto Y, Marusawa H, Kinoshita K, et al: Helicobacter pylori infection triggers aberrant expression of activation-induced cytidine deaminase in gastric epithelium. Nat Med 13: 470-476, 2007.

13. Lauren P: The two histological main types of gastric carcinoma: Diffuse and so-called intestinal-type carcinoma. An attempt at a histo-clinical classification. Acta Pathol Microbiol Scand 64 31-49, 1965.

14. Nakamura K, Sugano H and Takagi K: Carcinoma of the stomach in incipient phase: Its histogenesis and histological appearance. Gan 59: 251-258, 1968.

15. Egashira Y, Shimoda T and Ikegami M: Mucin histochemical analysis of minute gastric differentiated adenocarcinoma. Pathol Int 49: 55-61, 1999 .

16. Yoshino T, Shimoda T, Saito A, et al: Macroscopic features of differentiated adenocarcinoma with gastric or intestinal phenotype expression in early gastric cancer. Stomach Intestine 34: 513-525, 1999.

17. Koseki K, Takizawa T, Koike M, Ito M, Nihei Z and Sugihara K: Distinction of differentiated type early gastric carcinoma with gastric type mucin expression. Cancer 89: 724-732, 2000

18. Japanese Gastric Cancer Association: Japanese Classification of Gastric Carcinoma. 13th edition. Kanehara \& Co., Ltd., Tokyo, 1998. (In Japanese)

19. Schlemper RJ,Riddell RH,Kato Y, et al: The Vienna classification of gastrointestinal epithelial neoplasia. Gut 47: 251-255, 2000.

20. Dixon MF, Genta RM, Yardley JH and Correa P: Classification and grading of gastritis. The updated Sydney System. International Workshop on the Histopathology of Gastritis, Houston 1994. Am J Surg Pathol 20: 1161-1181, 1996.

21. Andachi H, Yashima K, Koda M, et al: Reduced Fhit expression is associated with mismatch repair deficiency in human advanced colorectal carcinoma. Br J Cancer 87: 441-445, 2002.
22. Baek MJ, Kang H, Kim SE, et al: Expression of hMLH1 is inactivated in the gastric adenomas with enhanced microsatellite instability. Br J Cancer 85: 1147-1152, 2001.

23. Niwa T, Ikehara Y, Nakanishi $\mathrm{H}$, et al: Mixed gastric- and intestinal-type metaplasia is formed by cells with dual intestinal and gastric differentiation. J Histochem Cytochem 53: 75-85, 2005.

24. Morisawa T, Marusawa H,Ueda Y, etal: Organ-specific profiles of genetic changes in cancers caused by activation-induced cytidine deaminase expression. Int J Cancer 123: 2735-2740, 2008.

25. Kim CJ, Song JH, Cho YG, et al: Activation-induced cytidine deaminase expression in gastric cancer. Tumour Biol 28: 333-339, 2007.

26. Takeda Y, Yashima K, Hayashi A, et al: Expression of AID, P53 and Mlh1 proteins in endoscopically resected differentiated-type early gastric cancer. World J Gastrointest Oncol 4: 131-137, 2012.

27. Goto A,Hirahashi M,Osada M,etal: Aberrantactivation-induced cytidine deaminase expression is associated with mucosal intestinalization in the early stage of gastric cancer. Virchows Arch 458: 717-724, 2011.

28. Pfeifer GP, Denissenko MF, Olivier M, Tretyakova N, Hecht SS and Hainaut P: Tobacco smoke carcinogens, DNA damage and p53 mutations in smoking-associated cancers. Oncogene 21: 7435-7451, 2002.

29. Saeki H, Ohno S, Miyazaki M, et al: p53 protein accumulation in multiple oesophageal squamous cell carcinoma: Relationship to risk factors. Oncology 62: 175-179, 2002.

30. Kozack R, Seo KY, Jelinsky SA and Loechler EL: Toward an understanding of the role of DNA adduct conformation in defining mutagenic mechanism based on studies of the major adduct (formed at $\mathrm{N}(2)-\mathrm{dG}$ ) of the potent environmental carcinogen, benzo[a]pyrene. Mutat Res 450: 41-59, 2000

31. Viala J, Chaput C, Boneca IG, et al: Nod1 responds to peptidoglycan delivered by the Helicobacter pylori cag pathogenicity island. Nat Immunol 5: 1166-1174, 2004.

32. Endo Y, Marusawa H, Kou T, et al: Activation-induced cytidine deaminase links between inflammation and the development of colitis-associated colorectal cancers. Gastroenterology 135: 889-898, 898.e1-898e.3, 2008.

33. Endo Y, Marusawa H, Kinoshita K, et al: Expression of activation-induced cytidine deaminase in human hepatocytes via NF-kappaB signaling. Oncogene 26: 5587-5595, 2007.

34. Komori J, Marusawa H, Machimoto T, et al: Activation-induced cytidine deaminase links bile duct inflammation to human cholangiocarcinoma. Hepatology 47: 888-896, 2008.

35. Nagata N, Akiyama J, Marusawa H, et al: Enhanced expression of activation-induced cytidine deaminase in human gastric mucosa infected by Helicobacter pylori and its decrease following eradication. J Gastroenterol 49: 427-435, 2014.

36. Kang DW, Hwang WC, Park MH, et al: Rebamipide abolishes Helicobacter pylori CagA-induced phospholipase D1 expression via inhibition of NFKB and suppresses invasion of gastric cancer cells. Oncogene 32: 3531-3542, 2013.

37. Sugai T, Habano W, Endoh M, et al: Molecular analysis of gastric differentiated-type intramucosal and submucosal cancers. Int J Cancer 127: 2500-2509, 2010.

38. Fritz EL and Papavasiliou FN: Cytidine deaminases: AIDing DNA demethylation? Genes Dev 24: 2107-2114, 2010.

39. Tajima Y, Shimoda T, Nakanishi Y, et al: Gastric and intestinal phenotypic marker expression in gastric carcinomas and its prognostic significance: Immunohistochemical analysis of 136 lesions. Oncology 61: 212-220, 2001.

40. Tsukashita S, Kushima R, Bamba M, Sugihara H and Hattori T: MUC gene expression and histogenesis of adenocarcinoma of the stomach. Int J Cancer 94: 166-170, 2001.

41. Yuasa H, Inada K, Watanabe $H$ and Tatematsu M: A phenotypic shift from gastric-intestinal to solely intestinal cell types in intestinal metaplasia in rat stomach following treatment with X-rays. J Toxicol Pathol 15: 85-93, 2002. 